\title{
AN NOVEL NEURAL NETWORK TRAINING BASED ON HYBRID DE AND BP
}

\author{
Xiaohui Yuan ', Yanbin Yuan ${ }^{2}$, Cheng Wang ${ }^{1}$ \\ 1 Huazhong University of Science \& Technology, 430074 Wuhan, China 2 Wuhan University \\ of Technology, 430071 Wuhan, Chnia
}

Abstract: This paper proposes a new approach for training FNN by hybrid DE and BP. It combines the advantages of the global search performed by DE over the FNN parameter space and the local search of BP. Using a function approximation as an illustration, we compare the HDEBP and BP for effectiveness and efficiency for training FNN. It shows that the use of new method can provide better results than BP.

Key words: neural networks, differential evolution, BP algorithm

\section{INTRODUCTION}

It has been shown that multilayer feed-forward networks(FNN) can be used with great success to solve nonlinear optimization problems. The main difficulty in using FNN is the training phase, which can be error prone and slow, due to its nonlinear nature. Supervised NN training has been widely researched and many optimization algorithms have been devised, most of which have been based on the gradient descent techniques. Typically a variation of $\mathrm{BP}$ have been developed to train them, such as the gradient descent BP and Levenberg-Marquardt BP etc.

However, as pointed out by Curry and Morgan ${ }^{[1]}$, BP based on gradient algorithm do not provide the best and fastest way to train NN, because $\mathrm{NN}$

This work was supported by National Natural Science Foundation of China under Grant No. 50409010 \& 50309013 
generate complex error surfaces with multiple local optima, even for simple functions being estimated, $\mathrm{BP}$ tends to become trapped in local solutions that are not global. Since BP converges locally, the solutions found by this gradient technique are highly dependent on the initial random draw of weights, which are rather sensitive to their initial weights in the error space. Therefore, it should be clear that the precise starting position could affect the speed and accuracy with which the algorithm will find the minimum. The problem of local minima can be efficiently avoided by using better initial weight values instead of randomly values. So one possibility could be a hybrid of global optimization algorithm that provides the best initial weight values and BP method for training FNN.

DE is one of the recent population-based techniques. It was invented by K. Price and R. Storn in 1995 as an heuristic method for minimizing nonlinear and non-differentiable functions. It is successfully applied to many optimization problems, such as aerodynamic shape optimization, automated mirror design and mechanical engineering design.

To overcome BP local convergence for training $\mathrm{FNN}$, this paper proposed a new hybrid DE and BP(HDEBP) which used DE to initialize the weights values of FNN. Finally function approximation problem have showed the feasibility and effectiveness of the method for training FNN.

\section{DIFFERENTIAL EVOLUTION}

$\mathrm{DE}$ is a population-based algorithm that creates new individuals one at a time. It is a greedy algorithm in which the new individual replaces the poorest member provided the new individual outperforms it.

DE utilizes NP D-dimensional parameter vectors $x_{i, G},(i=1,2, \ldots N P$ as a population for each generation G). DE works as follows: First, all individuals are randomly initialized and evaluated using the fitness function. Afterwards, the following process will be executed as long as the termination condition is not fulfilled. DE generates new parameter vectors by adding the weighted difference between two population vectors to a third vector, which called mutation. The mutated vector's parameters are then mixed with the parameters of another predetermined vector, the target vector, to yield the trial vector, which called crossover. If the trial vector yields a lower cost function value than the target vector, the trial vector replaces the target vector in the following generation, which called selection. 


\section{Mutation}

For each target vector $\mathrm{x}_{\mathrm{i}, \mathrm{G}}$, a mutant vector is generated according to:

$$
v_{i, G+1}=x_{r 1, G}+F \cdot\left(x_{r 2, G}-x_{r 3, G}\right)
$$

where: $r_{1}, r_{2}, r_{3} \in\{1,2, \ldots N P\}$ are mutually different; $F$ is a real constant factor between $[0,2]$

\section{Crossover}

The trial vector $u_{i, G+1}=\left(u_{1 i, G+1}, u_{2 i, G+1}, \ldots u_{D i, G+1}\right)$ is formed according to:

$$
u_{j i, G+1}= \begin{cases}v_{j i, G+1} & \text { if }(\operatorname{rand}(j) \leq C R) \text { or } j=r n b(i) \\ x_{j i, G} & \text { if }(\operatorname{rand}(j)>C R) \text { and } j \neq r n b(i)\end{cases}
$$

where $\operatorname{rand}(j)$ is a uniform random number between $[0,1]$. CR is the crossover constant between $[0,1]$. $\mathrm{rnb}(\mathrm{i})$ is a randomly index from $1,2, . . \mathrm{D}$.

\section{Selection}

To decide whether or not it should become a member of generation $\mathrm{G}+1$, the trial vector $\mathrm{u}_{\mathrm{i}, \mathrm{G}+1}$ is compared to the target vector $\mathrm{x}_{\mathrm{i}, \mathrm{G}}$ using the greedy criterion. If vector $\mathrm{u}_{\mathrm{i}, \mathrm{G}+1}$ yields a smaller cost function value than $\mathrm{x}_{\mathrm{i}, \mathrm{G}}$, then $\mathrm{x}_{\mathrm{i}, \mathrm{G}+1}$ is set to $\mathrm{u}_{\mathrm{i}, \mathrm{G}+1}$; otherwise, the old value $\mathrm{x}_{\mathrm{i}, \mathrm{G}}$ is retained.

\section{THE FNN TRAINING BY HDEBP}

Since error surfaces for even simple problems can be quite complex with many local optima, the DE seems to be better suited for this type of search. It can be applied to global searches within the weight space of the FNN.

It is clear that the precise initial weights of the network can affect the speed and accuracy with which the BP will find the minimum. So this paper adopts DE training FNN to obtain optimal initial weights firstly. Then BP was used to train FNN, it can be furthermore improved significantly the performance for FNN training. This HDEBP works well for training FNN.

Output of a FNN is a function of synaptic weights $W$ and input values $x$, i.e. $y=f(x, W)$. In standard training processes, both the input vector $x$ and the output vector $\mathrm{y}$ are known, and the synaptic weights in $\mathrm{W}$ are adapted to obtain appropriate functional mappings from the input $\mathrm{x}$ to the output $\mathrm{y}$. Generally, the adaptation can be carried out by minimizing the network error function $\mathrm{E}$ which is of the form:

$$
E(y, f(x, W)):\left(y^{D 1}, x^{D 2}, W^{D 3}, f\right) \rightarrow R
$$


The optimization goal is to minimize function $\mathrm{E}(\mathrm{y}, \mathrm{f}(\mathrm{x}, \mathrm{W}))$ by optimizing the values of the network weights (now $D=D 3$ ):

$$
\mathrm{W}=\left(\mathrm{w}_{1}, \mathrm{w}_{2}, \ldots \mathrm{w}_{\mathrm{D}}\right)
$$

DE operates on a population $\mathrm{P}_{\mathrm{G}}$, of candidate solutions. These candidate solutions are the individuals of the population. DE maintains a population of constant size that consists of $\mathrm{NP}$, real-value vectors, $\mathrm{W}_{\mathrm{i}, \mathrm{G}}$, wher $\mathrm{i}$ is index to the population and $\mathrm{G}$ is the generation to which the population belongs.

$$
P_{G}=\left(W_{1, G}, W_{2, G}, \ldots W_{N P, G}\right)
$$

Additionally, in network training each vector contains D network weights:

$$
W_{i, G}=\left(W_{1, i, G}, W_{2, i, G}, \ldots W_{D, i, G}\right)
$$

For a FNN implementation, DE generates potential solutions using a forward propagation through FNN to obtain the value of the error function $E(y, f(x, W))$ for each individual in population at each generation. This error value is then used directly as the individual fitness. So, constructing an individual with a lower fitness is synonymous with training in the FNN. When the DE is finished, the global best individual serves as the high precise initial weight values of the FNN. Then we can use BP to train FNN directly. So, training performance of FNN can be improved significantly.

\section{EXPERIMENTAL RESULTS}

The HDEBP performance for training FNN is evaluated by experiment. In order to comparison of how well HDEBP and BP training FNN, function approximation problem was conducted using the sample data. The experiment was carried out using three-layer FNN and training function approximation problem, for example $y=\sin (x), x$ between $[0,2 \pi]$.

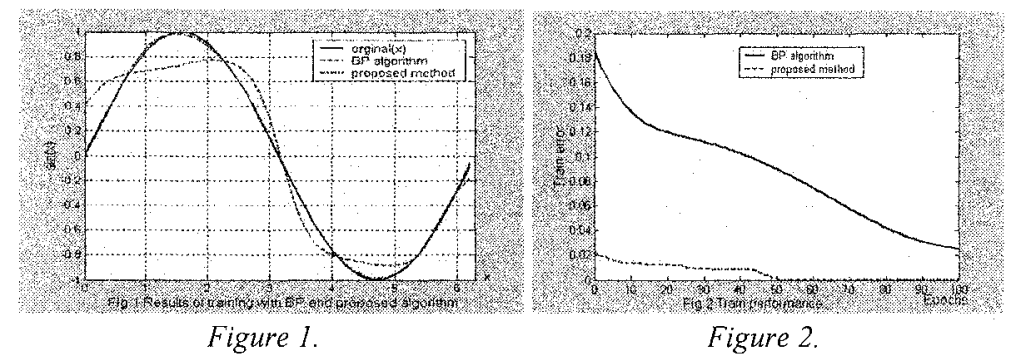

In the experiment, 63 sample points of $\sin (x)$ for $x=[0,2 \pi]$ and three layer FNN 1 - logsig(5)- pureline(1), where logsig(5) means 5 neurons with a sigmoid transfer function and pureline(1) means 1 neuron with a pure 
linear transfer function, were used. Both BP and HDEBP were applied to train the network with 5 hidden layer units. Fig 1 shows the original training data $(y=\sin (x))$ and network output after training with the BP and HDEBP. From Fig. 1, it is clear that HDEBP approximation effect is much higher than BP. This intuitively makes sense because BP may converge upon local solutions, while HDEBP can find global solutions.

The effectiveness of HDEBP was estimated by measuring the mean squared error for a training period of restricted max epochs 100 for sample. In the experiment, the HDEBP training was compared to BP. As shown in Fig2, we can find that HDEBP precision is much higher than BP. At the same time HDEBP had a faster convergence rate than BP. These comparisons also show that HDEBP captures the underlying function for the approximation problem rather very well. The FNN is trained by HDEBP, which speeds up convergence and overcomes local minima.

\section{CONCLUSIONS}

In this paper, a new HDEBP method based on hybrid DE and 'BP is proposed to train FNN. The performance of the HDEBP appeared to be comparable to that of $\mathrm{BP}$ for the function approximation, our simulation results show that the HDEBP is accurate and fast than BP for training FNN, which is much simpler to implement and yield overall better performance.

\section{REFERENCES}

1. Curry, B., Morgan, P.: Neural networks: a need for caution. Omega, International Journal of Management Sciences 25(1997)123-133

2. D. Patterson.: Artificial neural networks, theory and applications. Prentice Hall(1996)

3. P. Baldi.: Gradient descent learning algorithm overview: a general dynamical systems perpective. IEEE Trans. on neural networks,6(1)(1996)182-195

4. Moller m.: A scaled conjugate gradient algorithm for fast supervised learning. Neural networks, 6(1993)525-533

5. Hagan, M., Menhaj, M.: Training feed-forward networks with the Marquadt algorithm. IEEE Trans. on Neural networks,5(6)(1994)152-157

6. R., Storn, K., Price.: Differential Evolution - A Simple and Efficient Heuristic for Global Optimization over Continuous Spaces. Journal of global optimization, 1 1(1997)341-359 